\title{
Evaluation Of Phenotypic Methods For Detection Of Carbapenem Resistance In Isolates Of Pseudomonas Aeruginosa In A Tertiary Care Hospital
}

\author{
Shaista Bakhat, Yasmeen Taj, Faisal Hanif, Muhammad Faisal Faheem
}

ABSTRACT

Objective: To assess the effectiveness of Modified Hodge test (MHT) and Modified carbapenemase inactivation method (mCIM) for detection of carbapenemase enzyme produced by Pseudomonas aeruginosa strains that code for resistance towards carbapenem.

Study Design and Setting: This observational cross sectional study was carried out from January 18 to January 19 in microbiology department of PNS SHIFA Karachi.

Methodology: 140 isolates of Pseudomonas aeruginosa were cultured from pus samples of hospitalized patients from different wards like ENT, Surgery, Burn Unit, Plastic Surgery, ICU, Medicine, Pediatrics and family ward. These isolates were tested carbapenem resistance by two phenotypic methods namely MHT and mCIM test. This data was analyzed by using SPSS Version 23.0.

Results: In our research study mCIM method showed 100\% sensitivity, $99.1 \%$ specificity, positive predictive value $96.1 \%$ and negative predictive value $100 \%$ as compare to Modified Hodge Test which gave $77 \%$ sensitivity, $99.1 \%$ specificity, 95.2\% PPV and $100 \%$ NPV.

Conclusion: mCIM test is simple, accurate and more reliable method for detection of carbapenemase production as compared with MHT. It is recommended, cabapenemase producing isolates should be tested as a routine practice by all clinical labs laboratory.

Keywords: carbapenem resistance, mCIM, MHT, Pseudomonas aeruginosa, PPV, NPV.

\section{INTRODUCTION:}

The micro-organism Pseudomonas aeruginosa is a pathogen appearing as a common source of hospital acquired infections especially sepsis. ${ }^{1}$ Resistance of microorganisms towards antibiotics is an upcoming crucial challenge in treatment of infectious diseases. This resistance comes with undesirable outcomes and morbidity, prolonged hospitalization, expense and even mortality. ${ }^{2}$ Risk of death with Pseudomonas aeruginosa infection can surpass over $58.8 \%{ }^{3}$ After the superbug methicillin resistant staphylococcus aureus which causes infections both in hospital and community infections by Pseudomonas aeruginosa are occurring Worldwide with limited treatment choices as a multidrug resistance. Unfortunately these infections have limited treatment choices as they are multidrug resistant. Carbepenem is the last resort

- - - - - - - - - - - - - - - -

I Shaista Bakhat

Senior Lecturer, Pathology Department

I Bahria University Medical and Dental College, Karachi

I Email:shaistakhurram2@gmail.com

I Yasmeen Taj

I Professor, Pathology Department

I Bahria University Medical and Dental College, Karachi

I Faisal Hanif

Assistant Professor, Pathology Department

I Bahria University Medical and Dental College, Karachi

Muhammad Faisal Fahim

I Researcher and Consultant Statistician

I Bahria University College of Physical Therapy, Karachi

I Received: 07-03-2019

Accepted: 21-05-2019 against strains of Pseudomonas aeruginosa,${ }^{4}$ However its clinical use is facing problems as a result of resistance towards known carbapenem antibiotics (such as imipenem and meropenem). ${ }^{5}$ This resistance is multifactorial as overexpression of efflux system, production of enzymes, reduction of pore expression, reduction of external membrane proteins expressions and topoisomerase enzyme. Carbapenem resistance is also arbitrated by mutated genes that transcribed for enzymes carbapenemase. According to Ambler classification these genes are classified into four classes depending on the amino acid sequence. Class A (KPC) and D (OXA) act through a serine-based mechanism while class B (IMP, VIM) depends on zinc to work therefore are called metallo-beta-lactamase. KPC [Klebsiella pneumonae carbapenemase] and VIM [Verona integron-encoded metallobeta-lactamase] were regarded as the numerous type of carbapenemase. They were reported in United States, Israel, Turkey, China, India, the United Kingdom, Nordic countries and Greece till 2007. After 2008, NDM-1(New Delhi metallo beta-lactamase) was identified from isolates of Klebsiella pneumonae and $E$ coli from India and Pakistan. OXA-48 genes are other plasmid borne which transcribe resistance and have been detected in strains of Pseudomonas aeruginosa from Middle East, Turkey and India.

Antibiotic resistant bacteria must be detected because suitable treatment is essential in curtailing the spread of resistant strains. Several phenotypic screening methods are used such as Kirby-Bauer (KB) disk diffusion antibiotic sensitivity test ${ }^{6}$ on Muller Hinton Agar (according to CLSI standards 
sensitive and resistant zone), Carba NP test and eCIM. Other phenotypic detection methods for carbapenemase production are Modified Hodge Test ${ }^{7}$ and mCIM method (modified carbapenemase inactivation). ${ }^{8}$ Regretfully, carbapenem resistance is hard to detect by $\mathrm{KB}$ disk diffusion method in different laboratories. Recent CLSI policies suggest carbapenemase screening as a standard constituent of routine laboratory work. Despite the availibility of molecular methods for detection of carbapenemases transcribing genes which are more accurate they cannot be used for routine laboratory work as the method cumbersome and expensive. Among number of phenotypic used methods easily available are MHT and mCIM methods. MCIM method has newly been introduced by CLSI [http://clsi.org/standards/micro/ microbiology files] $]^{9}$ for phenotypic detection of carbapenemase in Enterobacteriaceae ${ }^{10}$. The efficacy of this test was assessed in a multicenter test and found to have $97 \%$ mean sensitivity and 99\% specificity. ${ }^{11}$ Another study carbapenemase was detected in Pseudomonas aeruginosa by mCIM and PCRdetected genes (KPC, GES, IMP, VIM, NDM, OXA-48, and NMC/IMI). ${ }^{8}$

Detection of carbapenemase in Pseudomonas aeruginosa strain is of extreme significance to evade hospital acquired resistant infections. The accessibility of precise and inexpensive carbapenemase detection methods may be an inducement for laboratories to scrutinize this problem and help prevent a major threat of antibiotic resistance trend in bacteria.

\section{METHODOLOGY:}

The observational cross sectional study was done at PNS Shifa a tertiary care hospital, Karachi. 140 samples of Pseudomonas aeruginosa were collected from jan 2018jan 2019. This study was approved by Ethical Review Committee of PNS Shifa. Informed consent was taken from hospitalized patients. The strains of Pseudomonas aeruginosa were isolated from pus samples of infection of different body parts. Out-door patients, repeat samples from same patients and patients already on antibiotics were excluded. Samples of $P$. aeruginosa were received from various wards (Burn unit, ENT ward, Plastic surgery ward, paedriatic ward, family ward and ICU). Strains of Pseudomonas aeruginosa were grown on blood agar ${ }^{12}$, MacConkey agar ${ }^{13}$. Pseudomonas aeruginosa clinical isolates yield large, smooth, mucoid colonies with flat edges and green pigments (Guangzhou Ikeme technology co Ltd) after incubation at $35^{\circ} \mathrm{C} \pm 2^{\circ} \mathrm{C}$ for 24 hours. They gave colourless colonies on MacConkey agar (Shanghai Hungsun chemical co Ltd) after incubation at $35^{\circ} \mathrm{C} \pm 2^{\circ} \mathrm{C}$ for 24 hours. Gram staining was done for confirmation of Gram negative rods. Then biochemical test (oxidase test) (Scien Cell) was done for Pseudomonas aeruginosa. When the reagent was oxidized by cytochrome $\mathrm{C}$, it changed from colorless to a dark blue or purple compound, indophenols blue.
According to standard guidelines, preliminary AST (antibiotic susceptibility test) screening was done with disc diffusion method. Antimicrobial susceptibility testing was checked by disc diffusion method on Mueller -Hinton (MH) agar plate (Oxoid CM0337) as per CLSI 2019 (Clinical laboratory standard international) guidelines. Two carbapenem antibiotics like Meropenem $10 \mu \mathrm{g}$ (oxoid company) and Imipenem $10 \mu \mathrm{g}$ (Oxoid ) were used. When zone is equal or less than $15 \mathrm{~mm}$ it is interpreted as resistance both for imipenem and meropenem. Lawning was prepared with a 1:10 dilution of a $0.5 \mathrm{McF}$ arland suspension of Escherichia coli ATCC 25922 on Muller Hinton Agar. Diameter of zone is $16-18 \mathrm{~mm}$ is considered as intermediate. Diameter is equal or more than 19 indicates sensitivity for imipenem and meropenem.

Modified Hodge test and mCIM test were performed in all cases. Mueller Hinton agar was used to perform modified Hodge test ${ }^{14}$. Lawning was done with a 1:10 dilution of a $0.5 \mathrm{McF}$ arland suspension of Escherichia coli ATCC 25922(provided by microbiology department of PNS shifa) on Muller Hinton Agar. Meropenem 10 $\mu$ g was used. Then test organisms and control positive were streaked on the lawn in a straight line from the edge of disc to the edge of the plate. These Mueller Hinton agar plates were incubated at $35 \pm 2^{\circ} \mathrm{C}$ for 24 hours. After 16-24 hours of incubation, a clover-leaf-type indentation was examined at the intersection of the test organism and E coli 25922, within the zone of inhibition of the carbapenem susceptibility disk. MHT (modified Hodge test) positive test indicated the carbapenemase production.

$\mathrm{mCIM}^{15}$ was also used for phenotypic detection of carbapenemase. With the help of sterile inoculating loop, $1 \mu 1$ of test organism was put into $2 \mathrm{ml}$ tube of tryptic soy broth (TSB, Hi Media Laboratories); suspension was vortexed for $10-15 \mathrm{secs}$. Then $10 \mu \mathrm{g}$ MEM disk was placed into $2 \mathrm{ml}$ tube and incubated for 4 hours \pm 15 minutes at $35^{\circ} \mathrm{C} \pm 2{ }^{\circ} \mathrm{C}$.

E coli ATCC 25922 with turbidity equivalent to a 0.5 McFarland was prepared. Lawning was done on Mueller Hinton Agar. The MEM (meropenem) disk was removed from the TSB suspension and placed it on MHA plate and incubated for 24 hours. Results are considered as positive with zone $6-10 \mathrm{~mm}, 11-19 \mathrm{~mm}$ zone an intermediate results and $>20 \mathrm{~mm}$ as negative (no carbapenemase detected).

Statistical analysis of the data was done using SPSS version 23.0. Results were reported as frequencies (percentages) for categorical variables i.e source of specimens. The sensitivity and specificity of MHT for detection of carbapenemaseproducing Pseudomonas aeruginosa were calculated and compared with mCIM as the standard method by chi square method. P-value $<0.05$ is considered as statistically significant.

\section{RESULTS:}

140 isolates of Psedomonas aeruginosa were cultured from 
pus samples of different infection sites. The most frequent isolates were from ENT infections (47.1\%) followed by co surgical wound infections (13.6\%), burns super-infections (12.1\%), diabetic foot $(8.6 \%)$, plastic post-operative infections (7.9\%), pediatric wound infections (1.4\%) and officer ward $(0.7 \%)$ as shown in (Tab 1). According to KB Disc Diffusion method, out of 140 Pseudomonas aeruginosa isolates 17 had zone size of $=15 \mathrm{~mm}$ indicating resistance according to CLSI 2019 as per Table 2. The results of mCIM showed 25 cases out of 140 as resistant with $100 \%$ sensitivity, $99.1 \%$ specificity, $96.1 \%$ positive predictive value and $100 \%$ negative predictive value as per Table 3. The results of MHT showed 20 resistant cases with $77 \%$ sensitivity, $99.1 \%$ specificity, $95.2 \%$ positive predictive value and $100 \%$ negative predictive value as per Table 4 .

\section{DISCUSSION:}

Pseudomonas aeruginosa is the causative microorganism of hospital acquired infections. Multiple factors are

Table 1: Frequency of infection sites

\begin{tabular}{|l|c|c|}
\hline Ward & Frequency & Percent \\
\hline ENT & 66 & 47.1 \\
\hline Surgery & 19 & 13.6 \\
\hline Burn Unit & 17 & 12.1 \\
\hline 10-ICU & 12 & 8.6 \\
\hline Medicine & 12 & 8.6 \\
\hline Plastic Surgery & 11 & 7.9 \\
\hline Paedriatics & 2 & 1.4 \\
\hline officer ward & 1 & 0.7 \\
\hline
\end{tabular}

Table 2: Antibiotic (Carbapenem) Susceptibility Test

\begin{tabular}{|c|c|c|c|}
\hline Carbapenems & $\begin{array}{c}\text { Resistant } \\
<=\mathbf{1 5} \mathbf{~ m m}\end{array}$ & $\begin{array}{c}\text { Intermediate } \\
\mathbf{1 6 - 1 8 \mathbf { m m }}\end{array}$ & $\begin{array}{c}\text { Sensitive } \\
>=\mathbf{1 9} \mathbf{~ m m}\end{array}$ \\
\hline \multirow{2}{*}{ IMP } & 17 & 0 & 123 \\
\cline { 2 - 4 } & $12 \%$ & $0 \%$ & $88 \%$ \\
\hline \multirow{2}{*}{ MEM } & 17 & 0 & 123 \\
\cline { 2 - 4 } & $12 \%$ & $0 \%$ & $88 \%$ \\
\hline
\end{tabular}

Table 3: $2 \times 2$ contingency table for mCIM

\begin{tabular}{|c|c|c|c|}
\hline \multirow{2}{*}{} & \multicolumn{2}{|c|}{ mCIM } & \\
\cline { 2 - 4 } & Positive & Negative & Total \\
\hline Positive & 25 & 1 & 26 \\
\hline Negative & 0 & 114 & 114 \\
\hline Total & 25 & 115 & 140 \\
\hline
\end{tabular}

Table 4: $2 \times 2$ contingency table for MHT

\begin{tabular}{|c|c|c|c|}
\hline \multirow{2}{*}{} & \multicolumn{2}{|c|}{ MHT } & \\
\cline { 2 - 4 } & Positive & Negative & Total \\
\hline Positive & 20 & 1 & 21 \\
\hline Negative & 6 & 113 & 119 \\
\hline Total & 26 & 114 & 140 \\
\hline
\end{tabular}

responsible to make Pseudomonas aeruginosa as a nosocomial super bug such as imprudent administration of antibiotics, instrumentation and intrinsic resistance. So it is imperative to initiate suitable therapy. Several phenotypic methods are available for detection of carbapenemase producing microorganisms. Some clinical procedures for screening carbapenemase include MHT, CNPt, mCIM and eCIM. In our study, initial screening was done by AST. It was followed by two phenotypic methods MHT (Modified Hodge Test) and mCIM (Modified Carbapenemase Inactivation Method).

The most frequent Pseudomonas isolates from ENT samples as shown in tab 1. Other microbiological studies showed Pseudomonas aeruginosa is the most common cause of otitis media since II World War. The reason may be the common cold and ear infections after water infections. According to KB Disk Diffusion method out of 140 Pseudomonas aeruginosa isolates 17 had a zone size of $=15 \mathrm{~mm}$ indicating resistance according to CLSI 2019. $\mathrm{mCIM}$ showed 25 cases with zone diameter $=10 \mathrm{~mm}$. MHT showed 20 isolates having resistance towards meropenem and imipenem with clover leaf indentation. Our study showed that among the phenotypic methods mCIM had the highest sensitivity $100 \%, 99.1 \%$ specificity, $96.1 \%$ positive predictive value and $100 \%$ negative predictive value. While MHT showed low results with $77 \%$ sensitivity, $99.1 \%$ specificity, $95.2 \%$ positive predictive value and $100 \%$ negative predictive value. These findings indicate that resistant cases may be missed by KB Disk Diffusion method which is used routinely in laboratory.

According to CLSI, AST (Antibiotic susceptibility test) is used as screening for detection of carbapenem resistant Pseudomonas aeruginosa, but it is not a confirmatory test. ${ }^{16,17}$ Our findings in accordance with other studies like Van der Zwaluw et al (2015) recommended mCIM as highly sensitive, specific and inexpensive method for detection of carbapenemase. Virginia $M$ et al explained the effectiveness of mCIM (Modified Carbapenem Inactivation method) with $93 \%$ sensitivity and $100 \%$ specificity for phenotypic detection of carbapenemase production. ${ }^{18}$ Another study, Biewei Yu et al explained the effectiveness of mCIM for suspected carbapenemases among Enterobacteriaceae. ${ }^{19}$ These studies are in accordance with our studies and endorsed the findings of mCIM method.

In our study the finding of MHT with 77\% sensitivity, 99.1\% specificity, $95.2 \%$ positive predictive value and $100 \%$ negative predictive value is also in accordance with other studies in which Lee K, Lim YS and et al identified 67\% cases of metallo-beta-lactamase producing organisms with MHT. ${ }^{20}$ After that Modified Hodge Test was introduced a step ahead of simple hodge test. A study was done in Pakistan explained the effectiveness of Modified Hodge Test with $69 \%$ detection of carbapenemases. ${ }^{21}$ But with passage of time MHT had lost its effectiveness especially in detecting 
NDM (New Dehli metallo-beta-lactamase). So Fernando Pasteron et al used Triton X-100 in their study in order to enhance the effectiveness of MHT for detection of NDM. ${ }^{22}$ NDM-1 is zinc dependent enzyme,they considered deficiency of zinc in MHT was responsible for poor detection of NDM1 . They added $100 \mathrm{microg} / \mathrm{ml}$ zinc sulfate but still failed. NDM-1 is lipoprotein acchored to the outer membrane with presence of canonical lipidation sequence (LSGC), called the Lipodox. In Triton Hodge test,they added non ionic surfactant, which allowed the detection of membrane bound carbapenemase. This was followed by another study in 2018, PAE-MHT (Pseudomonas aeruginosa-Modified Hodge Test) and chrom ID carba agar for detection of carbapenemase. This study indicated that PAE-MHT and chrom ID carba are sensitive and specific. ${ }^{23}$ Further studies showed reduced value of this test for carbapenemase detection ${ }^{24}$ and endorced use of mCIM as a routine practice in laboratories. In our study high sensitivity positive predictive value (PPV) of mCIM supports the use of this test as a reliable tool for screening of carbapenemase producing strains of Pseudomonas aeruginosa. Prompt detection of carbapenem resistance is vital for infection control measures and epidemiological records. Further, it is important to make appropriate choice of antibiotics. Detection of carbapenem resistant Pseudomonas aeruginosa with simple and cost effective methods is recommended in absence of molecular techniques.

\section{CONCLUSION:}

mCIM test is simple, accurate and more reliable method for detection of carbapenemase production as compared with MHT. It is recommended, cabapenemase producing isolates should be tested as a routine practice by all clinical labs laboratory.

\section{REFERENCES:}

1. Mishra SN, Biswal SR, Pattnaik D, Detection of prevalence of metallo beta lactamases in clinical isolates of imipenem resistant Pseudomonas aeruginosa from neonatal septicemia cases in a tertiary hospital in Odisha, India. Int $\mathrm{J}$ contemp pediatr. 2018; 5(1):61-66.

2. Basak S, Singh P, Rajurkar M. Multidrug resistant and extensively drug resistant bacteria: A study. Journal of pathogens. 2016; ID 4065603

3. Nathwani D, Raman G, Sulham K, Gavaghan M, Menon V. Clinical and economic consequences of hospital-acquired resistant and multidrug-resistant Pseudomonas aeruginosa infections: a systematic review and meta-analysis. Antimicrob Resist Infect Control. BioMed Central.2016; 3(1):32.

4. Buehrle DJ, Shields RK, Clarke LG, Potoski BA, Clancy CJ, Nguyen MH. Carbapenem-resistant Pseudomonas aeruginosa bacteremia: risk factors for mortality and microbiologic treatment failure. Antimicrob agents chemoth. 2017; 61(1):e01243-16.

5. Sultan BA, Khan E, Hussain F, Nasir A, Irfan S. Effectiveness of modified Hodge test to detect NDM-1 carbapenemases: an experience from Pakistan. J Pak Med Assoc. 2013; 63(8):955-60.
6. Bagul US, Sivakumar SM, antibiotic susceptibility testing: A Review on current practices. Int pharm. 2016; 6(3):11-17

7. Amjad A, Mirza IA, Abbasi SA, Farwa U, Malik N, Zia F. Modified Hodge test: A simple and effective test for detection of carbapenemase production. Iran J microbiol. 2011; 3(4):189.

8. Lisboa LF, Turnbull L, Boyd DA, Mulvey MR, Dingle TC. Evaluation of a Modified Carbapenem Inactivation Method for Detection of Carbapenemases in Pseudomonas aeruginosa. $\mathrm{J}$ clin microbiol. 2018; 56(1):e01234-17.

9. http://clsi.org/standards/micro/microbiology files]

10. CLSI. 2017. Performance standards for antimicrobial susceptibility testing. CLSI supplement M100, $27^{\text {th }}$ ed Clinical and Laboratory Standards Institute, Wayne, PA.

11. Pierce VM, Simner PJ, Lonsway DR, Roe-Carpenter DE, Johnson JK, Brasso WB, Bobenchik AM, Lockett ZC, CharnotKatsikas A, Ferraro MJ, Thomson RB. The modified carbapenem inactivation method (mCIM) for phenotypic detection of carbapenemase production among Enterobacteriaceae. Journal of clinical microbiology. 2017: JCM-00193.

12. Golle A, Janezic S, Rupnik M. Low overlap between carbapenem resistant Pseudomonas aeruginosa genotypes isolated from hospitalized patients and wastewater treatment plants. PloS one. 2017; 12(10): 0186736.

13. Al-Ahmadi GJ, Roodsari RZ. Fast and specific detection of Pseudomonas Aeruginosa from other pseudomonas species by PCR. Annals of burns and fire disasters. 2016; 29(4):264.

14. Ibrahim AH, Al Fadhil AO. Phenotypic Detection of Carbapenemase-producing K. pneumoniae isolated from Patients attending Hospital Settings in Khartoum. ajmsc. 2018; 3(2).

15. Walthall K, Anderson K, Reese N, Lonsway D, Kamile Rasheed J, Karlsson M. Evaluation of the RAPIDEC CARBA NP, Conventional CarbaNP, and the Modified Carbapenem Inactivation Method (mCIM) Tests for Phenotypic Detection of Carbapenemase-Producing Organisms. AJCP. 2018; 150(suppl_1):S123-4.

16. CLSI. Performance standards for antimicrobial susceptibility testing-CLSI document M100-Ed 29. $29^{\text {th }}$ informational supplement-2019.

17. Buehrle DJ, Shields RK, Clarke LG, Potoski BA, Clancy CJ, Nguyen MH. Carbapenem-resistant Pseudomonas aeruginosa bacteremia: risk factors for mortality and microbiologic treatment failure. Antimicrob agents chemother. 2017; 61(1):e01243-16.

18. Pierce VM, Simner PJ, Lonsway DR, Roe-Carpenter DE, Johnson JK, Brasso WB, Bobenchik AM, Lockett ZC, CharnotKatsikas A, Ferraro MJ, Thomson RB. Modified carbapenem inactivation method for phenotypic detection of carbapenemase production among Enterobacteriaceae. J clin microbiol. 2017; 55(8):2321-33.

19. Yu B, Dong D, Wang M, Guo Y, Yin D, Hu F. Evaluation of modified carbapenem inactivation method for suspected carbapenemase among Enterobacteriaceae clinical isolates. Oncotarget. 2018; 9(49):29233

20. Lee K, Lim YS, Yong D, Yum JH, Chong Y. Evaluation of the Hodge test and the imipenem-EDTA double-disk synergy test for differentiating metallo-beta-lactamase-producing isolates of Pseudomonas spp. and Acinetobacter spp. J clin microbiol. 2003; 41(10):4623-9. 
Shaista Bakhat, Yasmeen Taj, Faisal Hanif, Muhammad Faisal Faheem

21. Amjad A, Mirza IA, Abbasi SA, Farwa U, Malik N, Zia F. Modified Hodge test: A simple and effective test for detection of carbapenemase production. Iranian journal of microbiology. 2011;3(4):189.

22. Pasteran F, Gonzalez LJ, Albornoz E, Bahr G, Vila AJ, Corso A. Triton Hodge test: improved protocol for modified Hodge test for enhanced detection of NDM and other carbapenemase producers. Journal of clinical microbiology. 2016;54(3):6409.
23. Othman HB, Halim RM, Abdul-Wahab HE, Atta HA, Shaaban O. Pseudomonas aeruginosa-Modified Hodge Test (PAEMHT) and ChromID Carba Agar for Detection of Carbapenemase Producing Pseudomonas Aeruginosa Recovered from Clinical Specimens. Open access Maced j Med Sci. 2018; 6(12):2283.

24. Ghasemnejad A, Doudi M, Amirmozafari NO. Evaluation of modified hodge test as a non-molecular assay for accurate detection of KPC-producing Klebsiella pneumoniae. Pol j microbiol. 2018; 67(3):291-5. 